\title{
Combining Numerous Uncorrelated MEMS Gyroscopes for Accuracy Improvement Based on an Optimal Kalman Filter
}

\author{
Honglong Chang, Member, IEEE, Liang Xue, Chengyu Jiang, Michael Kraft, and Weizheng Yuan
}

\begin{abstract}
In this paper, an approach to improve the accuracy of microelectromechanical systems (MEMS) gyroscopes by combining numerous uncorrelated gyroscopes is presented. A Kalman filter (KF) is used to fuse the output signals of several uncorrelated sensors. The relationship between the KF bandwidth and the angular rate input is quantitatively analyzed. A linear model is developed to choose suitable system parameters for a dynamic application of the concept. Simulation and experimental tests of a six-gyroscope array proved that the presented approach was effective to improve the MEMS gyroscope accuracy. The experimental results indicate that six identical gyroscopes with a noise density of $0.11^{\circ} / \mathrm{s} / \sqrt{ } \mathrm{Hz}$ and a bias instability of $62^{\circ} / \mathrm{h}$ can be combined to form a virtual gyroscope with a noise density of $0.03^{\circ} / \mathrm{s} / \sqrt{ } \mathbf{H z}$ and a bias instability of $16.8^{\circ} / \mathrm{h}$. The accuracy improvement is better than that of a simple averaging process of the individual sensors.
\end{abstract}

Index Terms-Array signal processing, filtering, gyroscope, microelectromechanical devices, random noise.

\section{INTRODUCTION}

$\mathbf{N}$ AVIGATION and guidance systems for small space or unmanned vehicles require compact inertial sensors, including gyroscopes and accelerometers, to provide position, velocity, and angular information about the vehicle [1]. The rapid development of microelectromechanical systems (MEMS) technology enables sufficiently small inertial sensors. However, to date, MEMS sensors are not accurate enough for such applications. Improving the resolution of MEMS inertial sensors has been a key issue in this field. The common methods usually focus on the design and fabrication of the sensing element by, e.g., having a high quality factor [2], ensuring good mode matching by active resonant frequency tuning [3],

Manuscript received December 9, 2011; revised March 2, 2012; accepted April 2, 2012. This work was supported in part by the Chinese National Science Foundation and in part by the Chinese New Century Excellent Talents in University. The Associate Editor coordinating the review process for this paper was Dr. George Xiao.

H. Chang is with the Micro and Nano Electromechanical Systems Laboratory and the Department of Microsystem Engineering, Northwestern Polytechnical University, Xi' an 710072, China, and also with the Micromachining Laboratory, California Institute of Technology, Pasadena, CA 91125 USA (e-mail: changhl@nwpu.edu.cn; changhl@mems.caltech.edu).

L. Xue, C. Jiang, and W. Yuan are with the Micro and Nano Electromechanical Systems Laboratory, Northwestern Polytechnical University, Xi' an 710072, China.

M. Kraft is with the School of Electronics and Computer Science, University of Southampton, SO17 1BJ Southampton, U.K.

Color versions of one or more of the figures in this paper are available online at http://ieeexplore.ieee.org.

Digital Object Identifier 10.1109/TIM.2012.2200818
[4], developing a high-aspect-ratio microfabrication process [5] to obtain a comparably heavy proof mass and high readout capacitances [7], and designing high-performance integrated readout and control electronics [8]-[10] which lower the noise introduced by the interface circuits, therefore improving the signal-to-noise ratio of the sensor system. Another fundamentally different but promising approach to improve the accuracy is to make use of the so-called virtual gyroscope technology [11]. Here, the term "accuracy improvement" is defined as the noise reduction for angular rate measurements [11] and is usually used to label the sensor performance improvement. This terminology is also adopted for this work. The virtual gyroscope relies on the fact that, with MEMS technology, the cost of individual sensors is low and they can be easily fabricated in large numbers on a single wafer. The fusion of several MEMS gyroscopes combined with signal processing algorithm made it possible to demonstrate significant improvements in measuring angular rate. This approach can further reduce noise, minimize bias instability, and improve overall accuracy beyond the accuracy limitations of individual gyroscopes. Bayard and Ploen first proposed to combine four separate MEMS gyroscopes to form a virtual gyroscope [11]; in particular, the relationship between the accuracy improvement and noise correlation was analyzed. The simulation results showed that four individual gyroscopes with a drift of $8.66^{\circ} / \mathrm{h}$ could be combined to a virtual gyroscope with a drift of $0.062^{\circ} / \mathrm{h}$ when the component gyroscopes have a correlation factor of -0.333 . In our previous work, a two-level optimal filtering scheme was designed to reduce the gyroscope bias instability through fusing a three-gyroscope array [12]. Stubberud and Stubberud discussed the method of averaging and filtering of multiple sensors to improve the accuracy [13]. Al-Majed and Alsuwaidan presented a scheme of a multifilter estimator where the noise correlation was used to improve the angular rate estimate, but no simulation or experimental results were presented [14].

These methods use a gyroscope array to combine multiple measurements of the same physical angular rate input signal to produce a virtual sensor. It is actually a multisensor fusion process of combining, comparing, and interpreting information between many homogeneous sensors [15]. The accuracy of the virtual sensor can effectively be improved by using an optimal estimate technique implemented as a Kalman filter (KF) for estimating and compensating the random noises of MEMS gyroscopes. 
The validity of the virtual gyroscope technology has been proved and illustrated by numerous simulations. A noise correlation between the individual gyroscopes in the sensor array is the basis and prerequisite for the virtual gyroscope to achieve significant accuracy improvements. However, in a practical implementation, a gyroscope array with a particular correlation factor is usually not possible to realize. On the contrary, the white noise of multiple separate MEMS gyroscopes is not correlated, as it is caused by thermal noise electronics. For the gyroscope array used in this work, we have calculated the correlation factors between the gyroscopes from measurement data, indicating that the correlation factors are smaller than 0.01 . Therefore, for all practical considerations, the sensors can be regarded as uncorrelated. This is the reason why virtual gyroscope systems only have been demonstrated in simulations to date.

For an array consisting of uncorrelated gyroscopes, there is a general viewpoint that optimal filtering results in an accuracy improvement equal to that of a simple averaging process; in other words, filtering of several uncorrelated sensors reduces the noise by the square root of the number of sensors. However, it should be pointed out that this is the case only for specific conditions, i.e., the bandwidth of the KF is much wider than that of the individual gyroscope in the array, e.g., $1 \mathrm{kHz}$ for $\mathrm{KF}$ and $40 \mathrm{~Hz}$ for the individual gyroscope. In this case, the bandwidth of the virtual gyroscope is nearly equal to that of the individual gyroscopes in the array. Under other conditions, as described in this paper, an improvement better than that of an averaging process can be obtained. We will describe and experimentally prove that the virtual gyroscope can perform better than a simple averaging process even if the individual sensors are uncorrelated.

This paper is organized as follows. In Section II, we explain the theoretical operation of the virtual gyroscope. In Section III, we analyze the virtual gyroscope. In Section IV, we describe the implementation of the virtual gyroscope in hardware. In Section V, we verify the accuracy improvement of the virtual gyroscope in detail through various simulations and experiments. In Section VI, we draw some conclusions.

\section{TheORETICAL Operation OF THE VIRTUAL GYROSCOPE}

The number of individual gyroscopes in the array can be chosen as any integer; for this work, $N=6$ was selected as a case study to construct a virtual gyroscope.

\section{A. State-Space Model for the Virtual Gyroscope}

To construct a virtual gyroscope, a gyroscope error model must be defined first. The model must correctly express the relationship between the input angular rates and the gyroscope outputs. In this paper, the errors considered in the error model were as in [16], which only includes white noise denoted as angular random walk (ARW) and bias drift due to rate random walk (RRW). The ARW and RRW are considered to be the most significant and dominant error sources even though some more complex models exist considering other nonideal effects such as scale factor error variations and misalignment [17], [18]. In the error model comprising only terms of ARW and RRW, the bias drift is regarded as the major noise term that will determine the accuracy of a MEMS gyroscope. To quantify the noise terms of a gyroscope, the Allan variance method is usually used to analyze the gyroscope data [19]. Therefore, we take a very popular model to describe the gyroscope as

$$
\left\{\begin{array}{l}
y_{i}=\omega+b_{i}+n_{i}, \quad i=1,2, \ldots, 6 \\
\dot{b}_{i}=w_{b i}
\end{array}\right.
$$

where $y_{i}$ is the output rate signal of the $i$ th gyroscope, $\omega$ is the true rate signal, $b_{i}$ is a slowly varying random quantity called the bias drift due to the RRW process $w_{b i}$, and $n_{i}$ is the white noise accounting for ARW.

As for the six-gyroscope array, (1) can be expressed in vector form as

$$
\left\{\begin{array}{l}
Y=H_{1} \cdot \omega+b+v \\
\dot{b}=w_{b}
\end{array}\right.
$$

with

$$
Y=\left[\begin{array}{c}
y_{1} \\
\vdots \\
y_{6}
\end{array}\right] \cdot v=\left[\begin{array}{c}
n_{1} \\
\vdots \\
n_{6}
\end{array}\right] \cdot b=\left[\begin{array}{c}
b_{1} \\
\vdots \\
b_{6}
\end{array}\right] \cdot w_{b}=\left[\begin{array}{c}
w_{b 1} \\
\vdots \\
w_{b 6}
\end{array}\right] \cdot H_{1}=\left[\begin{array}{c}
1 \\
\vdots \\
1
\end{array}\right]_{6 \times 1} .
$$

To design a complete state-space model for the KF, the true rate signal can be described by a specific model such as random walk or a first-order Markov process. In this paper, the true rate signal $\omega$ is modeled as random walk [11]; thus

$$
\dot{\omega}=n_{\omega} .
$$

Here, $n_{\omega}$ is assumed to be a zero-mean white noise with a variance $q_{\omega}$.

A KF is utilized to obtain estimates of the gyroscope bias drifts and true rate signal. The seven-element state vector is defined as

$$
X(t)=\left[\begin{array}{ll}
b^{T} & \omega
\end{array}\right]^{T}
$$

where $b$ is the bias drift vector of the gyroscope array and $\omega$ is the true rate signal. Using a $\mathrm{KF}$, based on the gyroscope error model (1) and the true rate signal model (4), the virtual gyroscope state-space model can be described as

$$
\left\{\begin{array}{l}
\dot{X}(t)=F \cdot X(t)+w(t) \\
Z(t)=H \cdot X(t)+v(t)
\end{array}\right.
$$

where $X(t)$ is the state vector. $Z(t)=Y$ is the vector of the output measurements of the gyroscope array. The KF coefficient matrix $F=0_{7 \times 7}$ and $H$ are given by

$$
H=\left[\begin{array}{ccccc}
1 & 0 & \cdots & 0 & 1 \\
0 & 1 & \cdots & 0 & 1 \\
\vdots & \vdots & \ddots & \vdots & \vdots \\
0 & 0 & \cdots & 1 & 1
\end{array}\right]_{6 \times 7} .
$$

In (6), both $w(t)$ and $v(t)$ are white noises that represent process noise and measurement noise, respectively. According 
to the true rate signal and random noise model of the gyroscope, they can be expressed as $w(t)=[w b, n \omega]^{T}$ and $v(t)=$ $[n 1, n 2, n 3, n 4, n 5, n 6]^{T}$ with

$$
\begin{aligned}
& \left\{\begin{array}{l}
E[w(t)]=0, \quad E\left[w(t) w^{T}(t+\tau)\right]=Q \delta(\tau) \\
E[v(t)]=0, \quad E\left[v(t) v^{T}(t+\tau)\right]=R \delta(\tau)
\end{array}\right. \\
& Q=\left[\begin{array}{cc}
Q_{b} & 0 \\
0 & q_{\omega}
\end{array}\right]
\end{aligned}
$$

where $Q$ and $R$ are the covariance matrices of the process noise $w(t)$ and measurement noise $v(t)$, respectively. $Q_{b}$ is the covariance matrix of the RRW vector $w_{b} . E[\bullet]$ is the mathematical expectation operator, and $\delta(\tau)$ is the Kronecker Dirac delta function. In particular, the matrices $Q_{b}$ and $R$ are not necessarily diagonal since the RRW and ARW noises of each gyroscope can be correlated with each other. Supposing that there are correlations in the gyroscope array, the correlated covariance matrix $Q_{b}$ can be expressed as

$$
Q_{b}=\left[\begin{array}{ccccc}
\sigma_{b 1}^{2} & \rho_{12} \cdot \sqrt{\sigma_{b 1}^{2} \sigma_{b 2}^{2}} & \cdots & \rho_{16} \cdot \sqrt{\sigma_{b 1}^{2} \sigma_{b 6}^{2}} \\
\rho_{21} \cdot \sqrt{\sigma_{b 2}^{2} \sigma_{b 1}^{2}} & \sigma_{b 2}^{2} & \cdots & \rho_{26} \cdot \sqrt{\sigma_{b 2}^{2} \sigma_{b 6}^{2}} \\
\vdots & \vdots & \ddots & \vdots \\
\rho_{61} \cdot \sqrt{\sigma_{b 6}^{2} \sigma_{b 1}^{2}} & \rho_{62} \cdot \sqrt{\sigma_{b 6}^{2} \sigma_{b 2}^{2}} & \cdots & \sigma_{b 6}^{2}
\end{array}\right]_{6 \times 6}
$$

where $\sigma_{b i}^{2}$ is the RRW noise variance associated with the $i$ th gyroscope in the array and $\rho_{i j}$ is the correlation factor between the $i$ th and $j$ th gyroscopes of the array. If a constant crosscorrelation $\rho$ exists between the identical component gyroscopes in the array with RRW noise variance $\sigma_{b}^{2}$, the matrix $Q_{b}$ can be expressed as

$$
Q_{b}=\sigma_{b}^{2} \cdot\left[\begin{array}{cccc}
1 & \rho & \cdots & \rho \\
\rho & 1 & \cdots & \rho \\
\vdots & \vdots & \ddots & \vdots \\
\rho & \rho & \cdots & 1
\end{array}\right]_{6 \times 6}
$$

\section{B. Optimal KF for Rate Estimation}

Based on the aforementioned state-space model (6), the continuous-time KF can be expressed as [20]

$$
\begin{aligned}
\dot{\hat{X}}(t) & =K(t)[Z(t)-H \hat{X}(t)] \\
K(t) & =P(t) H^{T} R^{-1} \\
\dot{P}(t) & =Q-P(t) H^{T} R^{-1} H P(t) .
\end{aligned}
$$

Equations (12)-(14) describe the relationship between the state vector $X(t)$, filter gain $K(t)$, and estimated covariance $P(t)$. It shows that the estimation of the state vector $X(t)$ can be obtained through solving a covariance differential equation (14), which is a matrix Riccati differential equation (RDE). To explain the accuracy improvement and reveal the inherent properties of a virtual gyroscope, an analytic solution was developed by Bayard and Ploen to solve the RDE, resulting in a steadystate filter gain $K_{\infty}$ [11]. Therefore, the optimal estimation of

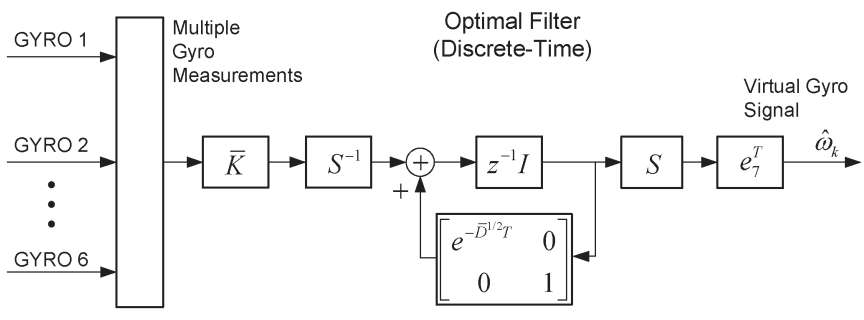

Fig. 1. Virtual gyroscope implementation using a discrete-time KF.

the true rate signal can be obtained by the following discretetime KF:

$$
\begin{aligned}
\hat{\zeta}_{k+1} & =\left[\begin{array}{cc}
e^{-\bar{D}^{\frac{1}{2}} T} & 0 \\
0 & 1
\end{array}\right] \hat{\zeta}_{k}+S^{-1} \bar{K} Z_{k} \\
\hat{\omega}_{k} & =e_{7}^{T} S \hat{\zeta}_{k}
\end{aligned}
$$

where $T$ is the sampling period, vector $e_{7}=[0,0, \ldots, 0,1]^{T}$, $\bar{K}$ represents the minimum-variance gain matrix, $\hat{\zeta}$ is the discrete-time filter state vector of dimension $N+1, \bar{D}$ is the diagonal matrix of the nonzero eigenvalues, and $\hat{\omega}_{k}$ is the optimal minimum-variance rate estimate, i.e., the virtual gyroscope output signal. The expressions of matrices $S, \bar{D}, K_{\infty}$, and $\bar{K}$ can be obtained from [11]. Thus, given multiple measurements of a six-gyroscope array, the output of the virtual gyroscope can be determined by the discrete-time KF described by (15) and (16). The implementation of a virtual gyroscope makes use of a discrete-time KF with a structure as shown in Fig. 1 [11]. The most important advantage with this approach is that it does not need to update the covariance $P(t)$ in each step, resulting in a reduced computational load for each sample as there are fewer calculations required for each iteration.

From the aforementioned description of state model and optimal KF, (7) holds for constant rate input, and (15) and (16) can be used to obtain a high-accuracy rate signal for the case of constant condition. For dynamic conditions, i.e., for time-varying rate inputs, the variance $q_{\omega}$ can be used to set an appropriate bandwidth to satisfy the dynamic requirement.

\section{ACCURACY OF The Virtual Gyroscope}

The accuracy of the virtual gyroscope signal can be evaluated by the covariance $P(t)$ since it represents the estimated variance of the state vector. Bayard and Ploen used an analytic solution to solve the problem of the matrix RDE [11]; in this way, an expression for the asymptotic covariance $P(t)$ associated with (14) was obtained. This analytic solution serves as the key for understanding all theoretical properties of the optimal filter and provides a complete characterization of the virtual gyroscope accuracy. From the asymptotic covariance $P(t)$, the covariance corresponding to the virtual gyroscope rate estimate can be obtained as $p_{\omega}(t)=e_{7}^{T} P(t) e_{7}$; thus, the drift of the virtual gyroscope can be obtained from $p_{\omega}(t)$ and expressed by [11]

$$
D_{\mathrm{vg}}=\frac{1}{H_{1}^{T} Q_{b}^{-1} H_{1}+1 / q_{\omega}}
$$




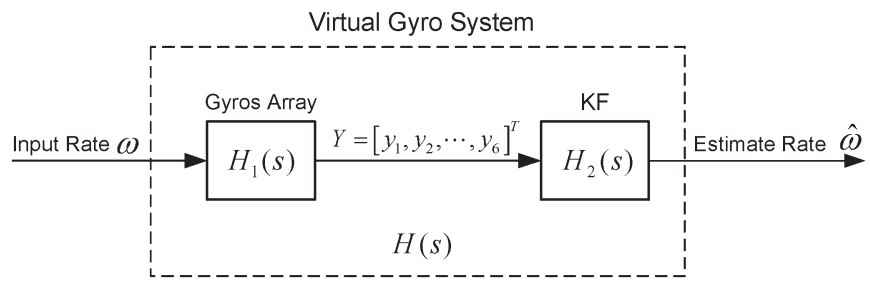

Fig. 2. Structure of transfer functions of virtual gyroscope system.

where $D_{\mathrm{vg}}$ is the drift of the virtual gyroscope. Given a gyroscope array with $N$ sensors, (17) indicates that the accuracy of the virtual gyroscope mainly depends on the parameter $q_{\omega}$ and the noise correlations between the component gyroscopes which are used to determine the system noise covariance matrix $Q_{b}$. Additionally, the true rate signal is modeled as a random walk with a noise variance $q_{\omega}$; hence, the dynamic properties of the virtual gyroscope (particularly the bandwidth) are a strong function of the parameter $q_{\omega}$.

The transfer function for the virtual gyroscope can be represented by the block diagram shown in Fig. 2. $H_{1}(s)$ and $H_{2}(s)$ are the transfer functions of the gyroscope array and the KF, respectively. It can be seen that a complete virtual gyroscope system is composed of the gyroscope array and the $\mathrm{KF}$. The bandwidth of the gyroscope array is equal to that of any individual gyroscope in the array, which can be regarded as a fixed value. The bandwidth of the KF is related to the parameters of KF such as variance $q_{\omega}$, number of individual gyroscopes $N$, and noise statistical quantities of the individual gyroscopes. Theoretically, the bandwidth of the virtual gyroscope system will be either smaller than or comparable to that of the individual gyroscope in the array. Therefore, we will discuss two cases for the accuracy of the virtual gyroscope with respect to bandwidth.

The first case is that, when setting the value of parameter $q_{\omega}$ to a very large value (or infinity), then we get $\left|H_{2}(j \omega)\right| \approx 1$; now, the bandwidth of the KF will become much larger than that of each gyroscope of the array. Therefore, the bandwidth of the virtual gyroscope is solely determined by the individual gyroscope in the array and will be comparable to that of the individual gyroscopes. For this case, substituting $q_{\omega} \rightarrow \infty$ into (17) results in

$$
D_{\mathrm{vg}}=\frac{1}{H_{1}^{T} Q_{b}^{-1} H_{1}}
$$

Assuming that a constant cross-correlation exists between the identical sensors of the array, inserting (11) into (18) yields

$$
D_{\mathrm{vg}}=\frac{1}{6} \sigma_{b}^{2}(1+5 \rho) \text {. }
$$

In this case, we can see that the accuracy of the virtual gyroscope is a function of the correlation factor $\rho$; (19) shows that the drift of the virtual gyroscope can be considerably reduced when the gyroscope array has a negative rather than a positive correlation factor. Furthermore, the best accuracy of the virtual gyroscope can be achieved if $\rho$ approaches $-1 / 5$. Most importantly, it is obvious that, in the uncorrelated situation, i.e., $\rho=0, \sqrt{\sigma_{b} / D_{\text {vg }}}$ is about $\sqrt{ } 6$; thus, the accuracy of the virtual

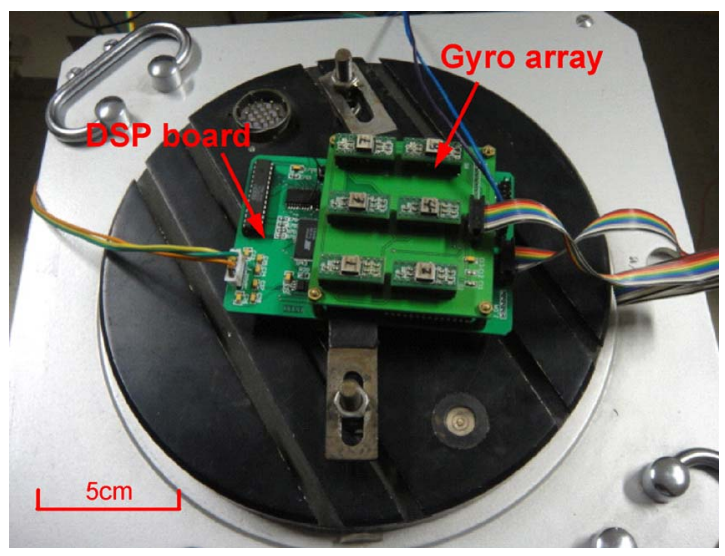

Fig. 3. Prototype of the virtual gyroscope system with six gyroscopes.

gyroscope is nearly equal to that of a simple averaging process. However, in any practical application, it is not necessary to set $q_{\omega}$ to infinity to guarantee the same bandwidth as the individual gyroscopes.

The second case is that the bandwidth of the KF is comparable to that of the individual gyroscopes in the array. When setting the value of $q_{\omega}$ to a specific value, then the bandwidth of the virtual gyroscope will be smaller than that of the individual gyroscopes. In this situation, the accuracy of the virtual gyroscope is a function of the value of $q_{\omega}$. For an uncorrelated situation, i.e., correlation factor $\rho=0$, inserting (11) into (17) results in

$$
D_{\mathrm{vg}}=\frac{q_{\omega} \sigma_{b}^{2}}{6 q_{\omega}+\sigma_{b}^{2}}
$$

Equation (20) indicates that the accuracy of the virtual gyroscope is related to the parameter $q_{\omega} . D_{\text {vg }}$ will become smaller with decreasing $q_{\omega}$. Furthermore, the bandwidth of the virtual gyroscope is heavily determined by the parameter $q_{\omega}$. Therefore, we can obtain an improvement beyond that of a simple averaging process through decreasing the bandwidth even though there is no correlation between the individual gyroscopes in the array. The relationship between the bandwidth and parameter $q_{\omega}$ will be quantitatively determined in the following.

\section{HARdwARE System IMPLEMENTATION}

The virtual gyroscope is mainly composed of a gyroscope array consisting of six separate gyroscopes, a central processing unit, an A/D data acquisition unit, a serial communication unit, and a Flash memory.

The prototype of the presented virtual gyroscope is shown in Fig. 3. Its overall size is about $10 \mathrm{~cm} \times 6 \mathrm{~cm}$; however, this could be reduced easily by a factor of ten if the electronics were to be implemented as an application-specific integrated circuit. Six MEMS gyroscopes ADXRS300 [21] are used to form a planar gyroscope array. Here, a DSP chip TMS320VC5416 [22] is chosen as the core processor for the virtual gyroscope. The A/D data acquisition unit uses 16-b ADS7807 to collect the voltage signals from the gyroscope array. The system operates in serial mode and connects with the DSP directly through the 16-b data bus. The bandwidth of each gyroscope is about $40 \mathrm{~Hz}$; 
thus, the sampling rate for the gyroscope array signal was set to $200 \mathrm{~Hz}$, which is five times of the bandwidth of the individual gyroscope. The Flash memory unit uses a 4-Mb Flash chip AM29LV400 to provide storage space for the system program. The virtual gyroscope output signal is transferred by a standard RS-232 interface.

\section{Results}

The relationship between the accuracy improvements and correlation factors was analyzed by simulations in [11]. As mentioned previously, currently, it is very difficult to obtain a gyroscope array with a negative correlation factor. In the following, the discussion about the virtual gyroscope is restricted to the case of uncorrelated sensors, i.e., $\rho=0$. In this section, simulation and experimental results will be presented to evaluate the accuracy of the virtual gyroscope. The major issues for a virtual gyroscope will be also analyzed and discussed.

\section{A. Simulations}

Before the simulations are described, some general rules are required to be formulated. First, the general unit for the variance $q_{\omega}$ is $\operatorname{deg}^{2} / \mathrm{h}^{3}$; here, the dimension of $\mathrm{deg}^{2} / \mathrm{h}^{2}$ is used to describe a normalized $q_{\omega}$ that can be interpreted as the rate variance in a 1-h period. Second, the standard deviation $(1 \sigma)$ of the estimated errors is used to evaluate the accuracy improvement of the rate signal before and after Kalman filtering in the dynamic condition; thus, the improvement factor is defined as

$$
I F=\frac{\sigma_{\text {Sgyro }}}{\sigma_{\text {Vgyro }}}
$$

where $I F$ is the improvement factor, $\sigma_{\mathrm{Sgyro}}$ is the $1 \sigma$ error for the single gyroscope in the array, and $\sigma_{\mathrm{Vgyro}}$ is the $1 \sigma$ value of the estimated errors for the virtual gyroscope signal. The $I F$ can be regarded as a normalized improvement factor compared to the single gyroscope before Kalman filtering.

1) Influence of $q_{\omega}$ on Accuracy Improvement: Through the aforementioned analysis, it was established that the accuracy of the virtual gyroscope mainly depends on the parameter $q_{\omega}$ when there is no correlation between the individual sensors. The parameter $q_{\omega}$ is difficult to determine and control since it is determined by the application environment and the dynamics of the input signal. However, the performance of the KF is strongly related to $q_{\omega}$; thus, in this section, different values of $q_{\omega}$ will be chosen to demonstrate the performance of KF and for comparison to a simple averaging process. Here, the relationship between the performance improvement for dynamic condition and parameter $q_{\omega}$ will be analyzed in detail by simulations.

The gyroscope array signals are generated by the gyroscope error model shown in (1) with a sampling rate of $200 \mathrm{~Hz}$, which is five times of the bandwidth of the individual gyroscope; the ARW and RRW noises are assumed as $2^{\circ} / \sqrt{ } \mathrm{h}$ and $240^{\circ} / \mathrm{h} / \sqrt{ } \mathrm{h}$, respectively. The true rate signal is assumed as a sinusoidal input signal $\omega=A * \sin (2 \pi f t)$, with an amplitude $A=10^{\circ} / \mathrm{s}$. Three different input frequencies are chosen, i.e., $f=0.1,0.3$, and $0.5 \mathrm{~Hz}$. By using the discrete-time filter methods, as shown in Fig. 1, and choosing different values of $q_{\omega}$, results of the

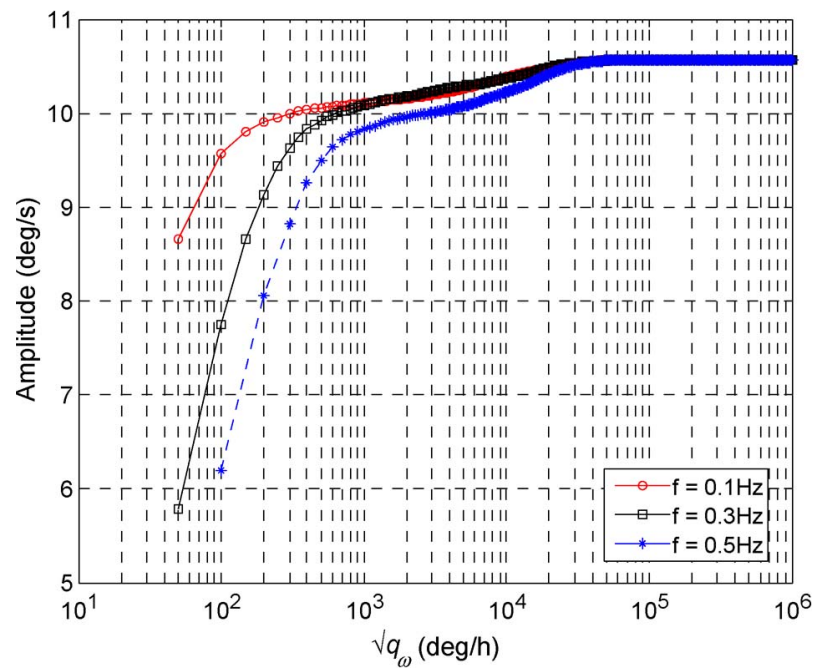

Fig. 4. Amplitude of virtual gyroscope signal filtered by different parameters $\sqrt{ } q_{\omega}$ for the input signals with various frequencies of $f=0.1,0.3$, and $0.5 \mathrm{~Hz}$.

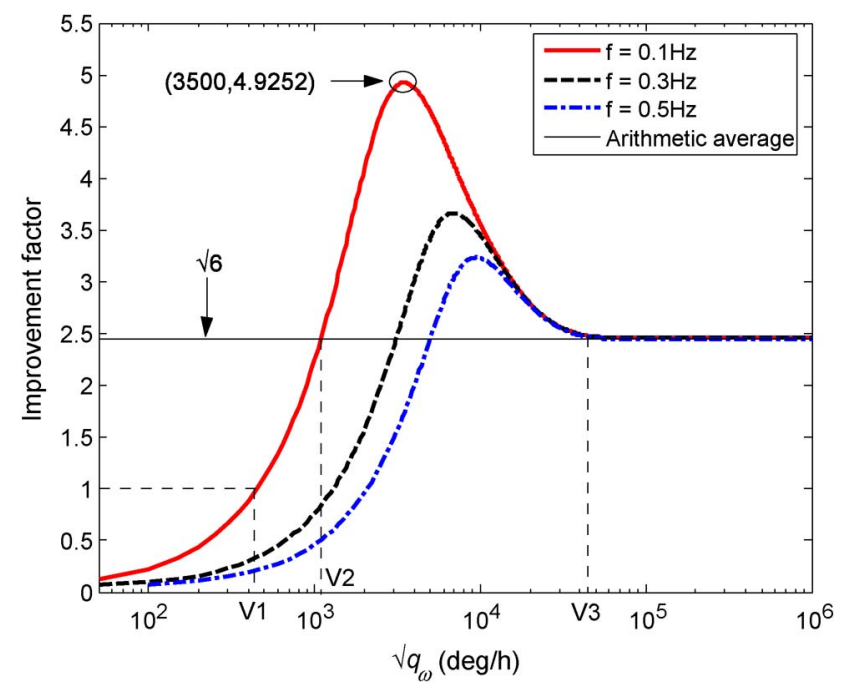

Fig. 5. Improvement factor of virtual gyroscope for different $\sqrt{ } q_{\omega}$ values, for three different input signals with frequencies of $f=0.1,0.3$, and $0.5 \mathrm{~Hz}$.

virtual gyroscope are obtained, as shown in Figs. 4 and 5, respectively.

From Fig. 4, it can be seen that the signal amplitude varies with $q_{\omega}$. In particular, when $q_{\omega}$ is small, the amplitude of the virtual gyroscope is considerably attenuated. For this case, the virtual gyroscope signal cannot accurately reproduce the dynamic behavior of the input rate signal. The signal amplitude increases and then reaches the amplitude of the true rate signal of $10^{\circ} / \mathrm{s}$ when $q_{\omega}$ is increased, and eventually reaches the amplitudes of the arithmetic average of $10.56^{\circ} / \mathrm{s}$ and $10.72^{\circ} / \mathrm{s}$ for different input frequencies. The relationship between the improvement factor and $q_{\omega}$ is also shown in Fig. 5. When $q_{\omega}$ is small, the improvement factor is lower than one; this implies that there are large attenuation and delay. Increasing $q_{\omega}$, the improvement factor becomes bigger and reaches the maximum value at the specified point corresponding to the optimal $q_{\omega}$. After the peak, the improvement begins to decline and eventually reaches the steady-state value, i.e., the averaging value of $\sqrt{ } 6$. 
This article has been accepted for inclusion in a future issue of this journal. Content is final as presented, with the exception of pagination.

TABLE I

DyNamic Simulation Results OF Virtual Gyroscope

\begin{tabular}{|c|c|c|}
\hline $\begin{array}{c}\text { Frequency } \boldsymbol{f} \\
(\mathbf{H z})\end{array}$ & $\begin{array}{c}\text { Optimal } \sqrt{\boldsymbol{q}_{\omega}} \\
(\% / \mathrm{hr})\end{array}$ & $\begin{array}{c}\text { Maximum } \\
\text { improvement factor }\end{array}$ \\
\hline 0.10 & 3500.0 & 4.93 \\
\hline 0.30 & 6900.0 & 3.67 \\
\hline 0.50 & 9500.0 & 3.24 \\
\hline
\end{tabular}

The optimal value of $q_{\omega}$ and the maximum improvement factor can be found from Table I.

We can see that different values of $q_{\omega}$ lead to different virtual gyroscope performances; the solid line, as shown in Fig. 5, corresponding to $f=0.1 \mathrm{~Hz}$ is selected to illustrate the principle as follows.

1) When $0<\sqrt{ } q_{\omega}<V 1$, the KF results in a considerable attenuation, and the virtual gyroscope signal cannot accurately reproduce the dynamic behavior of the input signal.

2) When $V 1<\sqrt{ } q_{\omega}<V 2$, the virtual gyroscope signal can reproduce the dynamic behavior of the input signal without attenuation; however, the KF performance is lower than that of a simple averaging process.

3) When $V 2<\sqrt{ } q_{\omega}<V 3$, the virtual gyroscope signal can reproduce well the dynamic behavior of the input signal without attenuation. Furthermore, the improvement factor is higher than $\sqrt{ } 6$, which means that the KF performance is higher than that of a simple averaging process. In particular, the improvement factor has a maximum value of about 4.93 for $\sqrt{ } q_{\omega}=3500^{\circ} / \mathrm{h}$; at this point, the KF shows optimum performance.

4) When $\sqrt{ } q_{\omega}>V 3$, the improvement factor approaches $\sqrt{ } 6$, and the KF degrades to that of a simple averaging process.

Now, some conclusions about the virtual gyroscope can be drawn. The virtual gyroscope can effectively work when the parameter $\sqrt{ } q_{\omega}$ is within $[V 1, \infty]$, and the best performance is obtained when the parameter $\sqrt{ } q_{\omega}$ is located in the range $[V 2, V 3]$. This means that a virtual gyroscope is inherently different from a simple averaging process even if the individual sensors are uncorrelated.

2) Influence of Input Frequency on Accuracy Improvement: From the aforementioned analysis, it can be seen that the maximum improvement factor and optimum working range will decrease with an increase in input frequency $f$. It also can be concluded that parameter $q_{\omega}$ corresponding to the maximum improvement factor should be increased as the input frequency increases.

The maximum improvement factor versus frequency of a sinusoidal input signal is plotted in Fig. 6. In this plot, each frequency corresponds to a specific $q_{\omega}$ for which the maximum accuracy improvement factor is determined, i.e., $q_{\omega}$ varies with frequency $f$. The graph indicates that the maximum improvement factor will decrease with increasing the input frequency and eventually approaches $\sqrt{ } 6$, i.e., the averaging effect. It also indicates that the slope of the improvement factor is larger for a lower input frequency such as $0-4 \mathrm{~Hz}$ and it is lower for higher frequencies. Moreover, in order to correctly reproduce the dynamic behavior of the input signal, the accuracy of the

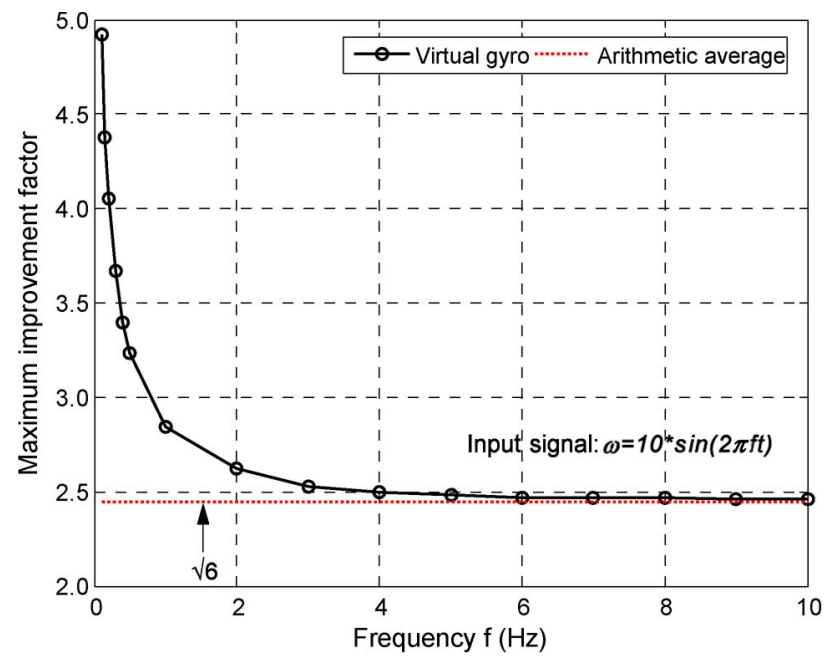

Fig. 6. Plot of maximum improvement factor versus the input signal frequency of the gyroscope array; $f$ ranges from 0.1 to $10 \mathrm{~Hz}$.

virtual gyroscope will approximate to that of an averaging process due to a larger value required for $q_{\omega}$.

From the aforementioned discussion, it can be seen that the parameter $q_{\omega}$ is crucial to the performance of the virtual gyroscope. Small $q_{\omega}$ easily results in attenuation of the gyroscope signal for a dynamic condition. A large $q_{\omega}$ degrades the performance of the KF to that of a simple averaging process. Therefore, choosing a proper $q_{\omega}$ value is a prerequisite for a successful application of this technique for a virtual gyroscope. The bandwidth will be used as a criterion to determine an appropriate value of $q_{\omega}$.

3) Relationship Between $q_{\omega}$ and KF Bandwidth: Due to the complex matrix computation required to solve the RDE, it is difficult to obtain an analytical expression for $q_{\omega}$ as a function of the bandwidth. Here, we use the KF frequency response to analyze this relationship and derive a simple linear expression. Based on the continuous-time KF described by (12) and steady-state filter gain $K_{\infty}$, the transfer function from the measurements to the virtual gyroscope signal can be expressed as

$$
\hat{\omega}(s)=e_{7}^{T}\left(s I+K_{\infty} H\right)^{-1} K_{\infty} Z(s) .
$$

The $-3-\mathrm{dB}$ standard is used to define the bandwidth of the KF. Using (22), with $\sqrt{ } q_{\omega}=10000^{\circ} / \mathrm{h}$, the frequency response of the KF is shown in Fig. 7. From the frequency response plot, it indicates a bandwidth of about $10.22 \mathrm{~Hz}$. The $-3-\mathrm{dB}$ bandwidths of the KF are presented in Table II for various values of $\sqrt{ } q_{\omega}$.

These results indicate that the $-3-\mathrm{dB}$ bandwidth will increase with increasing $q_{\omega}$. It can be found that the $-3-\mathrm{dB}$ bandwidth is linear with $\sqrt{ } q_{\omega}$, as shown in Fig. 8. Taking the 95\% confidence bounds, a linear fit model can be obtained as

$$
B W=0.001024 \times \sqrt{ } q_{\omega}-0.005483 .
$$

The root-mean-square error (RMSE) is only about 0.06 . Therefore, for applications in which the bandwidth characteristic can be determined, an appropriate value of $q_{\omega}$ for the virtual gyroscope can be easily determined from (23). 

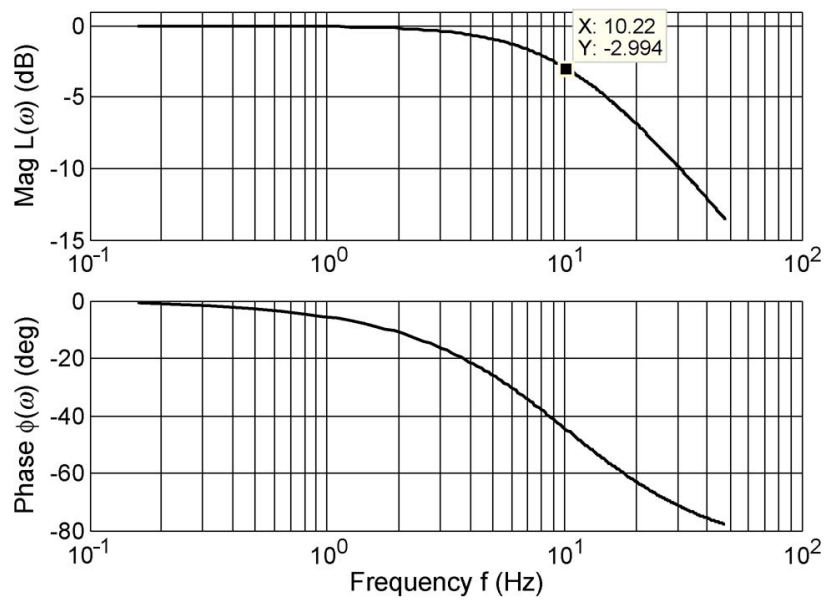

Fig. 7. Frequency response of the virtual gyroscope $\mathrm{KF}$ for $\sqrt{ } q_{\omega}=$ $10000^{\circ} / \mathrm{h}$.

TABLE II

-3-dB BANDWIDTH RESULTS OF KF

\begin{tabular}{|c|c|c|c|}
\hline $\begin{array}{c}\text { Parameter } \\
\sqrt{q_{\omega}}(\% / h r)\end{array}$ & $\begin{array}{c}\text { Bandwidth } \\
(\mathrm{Hz})\end{array}$ & $\begin{array}{c}\text { Parameter } \\
\sqrt{q_{\omega}}(\% / h r)\end{array}$ & $\begin{array}{c}\text { Bandwidth } \\
(\mathrm{Hz})\end{array}$ \\
\hline 2000.0 & 2.08 & 20000.0 & 20.44 \\
\hline 6500.0 & 6.71 & 25000.0 & 25.55 \\
\hline 8000.0 & 8.14 & 30000.0 & 30.82 \\
\hline 10000.0 & 10.22 & 35000.0 & 35.77 \\
\hline 15000.0 & 15.33 & 38000.0 & 38.96 \\
\hline
\end{tabular}

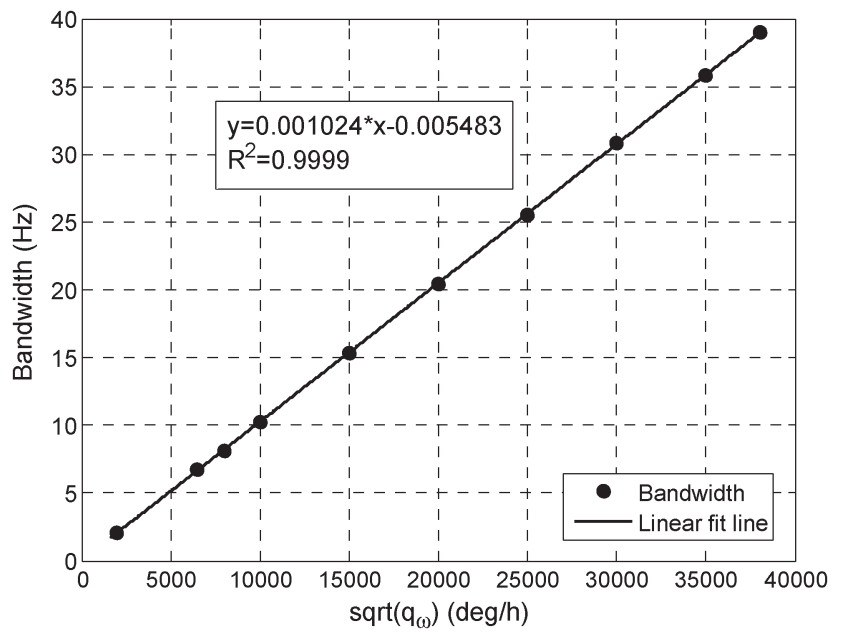

Fig. 8. Results of the $-3-\mathrm{dB}$ bandwidth of a virtual gyroscope $\mathrm{KF}$ as a function of parameter $\sqrt{ } q_{\omega}$.

\section{B. Experiments}

From the aforementioned analysis, it can be seen that, when the parameter $q_{\omega}$ approaches infinity, the accuracy of the virtual gyroscope can be regarded as that of an averaging process; in this case, the bandwidth of the virtual gyroscope reaches its maximum. However, if the bandwidth is reduced, an improvement better than that of the averaging process can be obtained. In this section, we present experimental results quantifying the accuracy of the virtual gyroscope.

The scale factor, bias drift, and noise density of the virtual gyroscope system were tested. The noises of the virtual gyroscope were evaluated using fast Fourier transform (FFT)

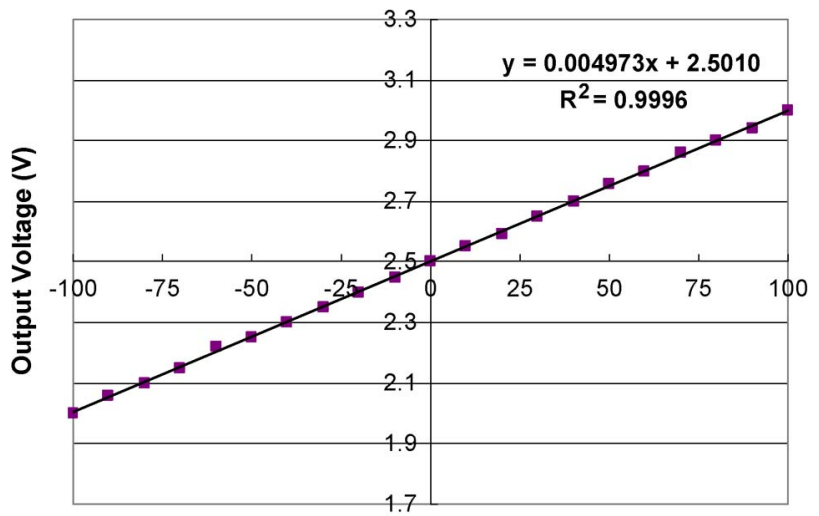

Angular Rate Input (deg/s)

Fig. 9. Scale factor of the virtual gyroscope system in $B W$ of $15 \mathrm{~Hz}$.

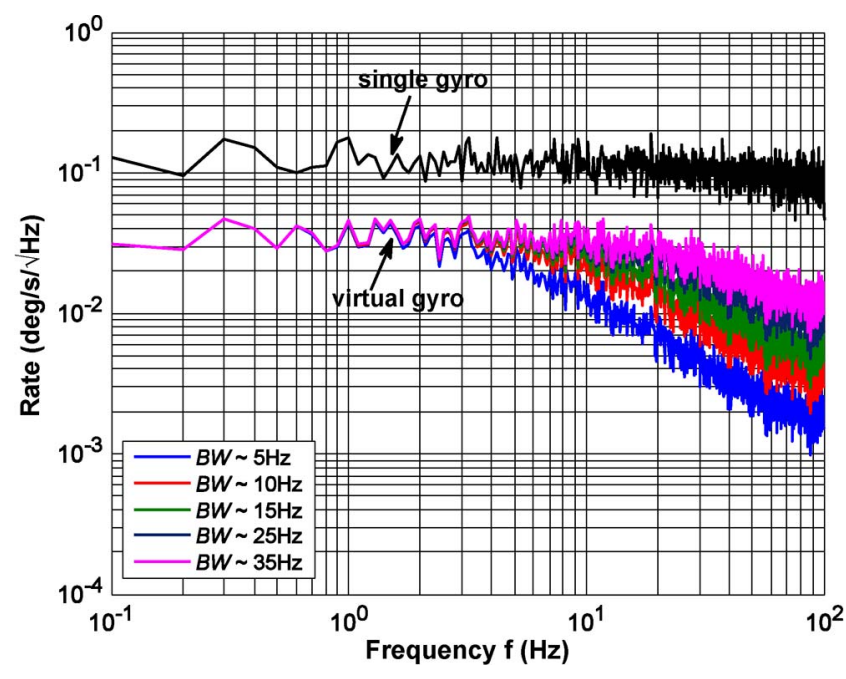

Fig. 10. FFT plot of the virtual gyroscope and the component gyroscopes.

analysis and root Allan variance of a zero rate output recorded for $1 \mathrm{~h}$ at a sampling rate of $200 \mathrm{~Hz}$. The signals of the virtual gyroscope were analyzed under different KF bandwidths (5, 10, 15 , and $25 \mathrm{~Hz}$ ), where the corresponding values of $q_{\omega}$ were chosen according to the bandwidth fit equation (23).

The relationship between the outputs of the virtual gyroscope under different input angular rates is shown in Fig. 9. With a full range of $300^{\circ} / \mathrm{s}$, the virtual gyroscope has a sensitivity of $4.973 \mathrm{mV} / \%$ s and the nonlinearity is $0.01 \%$, while the specification value of an individual gyroscope given by the manufacturer is $5.0 \mathrm{mV} / \% / \mathrm{s}$.

From the FFT plot of the rate signal (Fig. 10), the noise level indicates noise floor values of $\sim 0.11^{\circ} / \mathrm{s} / \sqrt{ } \mathrm{Hz}$ above $1 \mathrm{~Hz}$ for the single gyroscope in the array and $0.03^{\circ} / \mathrm{s} / \sqrt{ } \mathrm{Hz}$ for the virtual gyroscope. Here, $q_{\omega}$ is fixed for a given bandwidth, i.e., it does not change with frequency. A comparison of Allan variance measurement between the best single gyroscope in the array and the virtual gyroscope is shown in Fig. 11; the detailed results are illustrated in Table III. From the Allan variance plot, the ARW of the virtual gyroscope is revealed to be about a mean value of $1.56^{\circ} / \sqrt{ } \mathrm{h}$ for different bandwidths. The bias instability is observed to be $16.81^{\circ} / \mathrm{h}$, while the corresponding values of the best single gyroscope in the array are $6.19^{\circ} / \sqrt{ } \mathrm{h}$ and $61.99^{\circ} / \mathrm{h}$, respectively. 


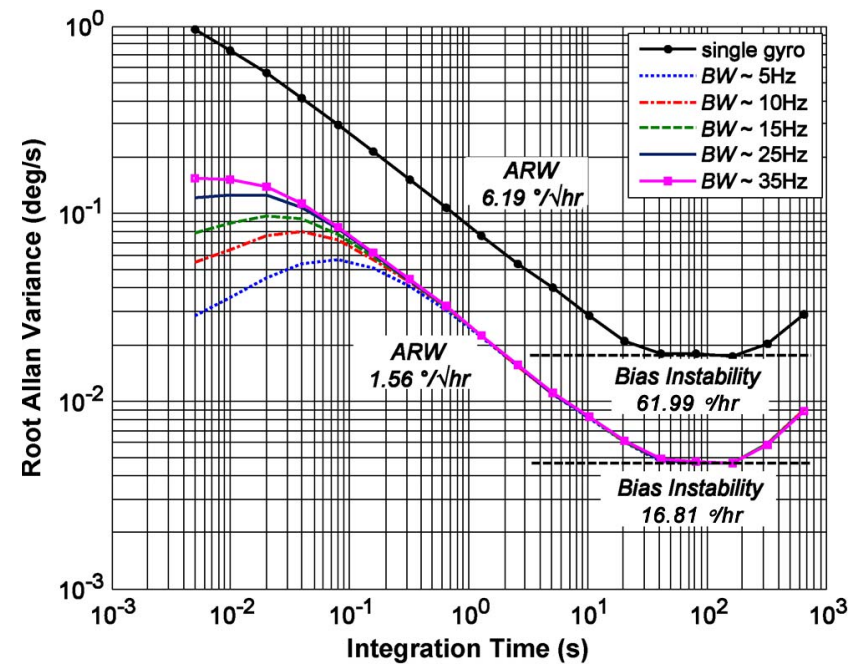

Fig. 11. Allan variance test results of the virtual gyroscope and component gyroscopes.

TABLE III

Performance of Designed Virtual Gyroscope System

\begin{tabular}{|c|c|c|c|c|c|}
\hline Bandwidth ( $\mathrm{Hz}$ ) & 5 & 10 & 15 & 25 & 35 \\
\hline $\begin{array}{c}\text { Noise Density } \\
(\% / \sqrt{ } H z)\end{array}$ & 0.03 & 0.03 & 0.03 & 0.03 & 0.03 \\
\hline$A R W(\% \sqrt{ } h r)$ & 1.51 & 1.54 & 1.55 & 1.59 & 1.60 \\
\hline Bias Drift $(\% / h r)$ & 16.71 & 16.79 & 16.80 & 16.85 & 16.88 \\
\hline$R R W(\% / h r / \sqrt{ } h r)$ & 126.69 & 126.51 & 125.79 & 125.14 & 125.04 \\
\hline
\end{tabular}

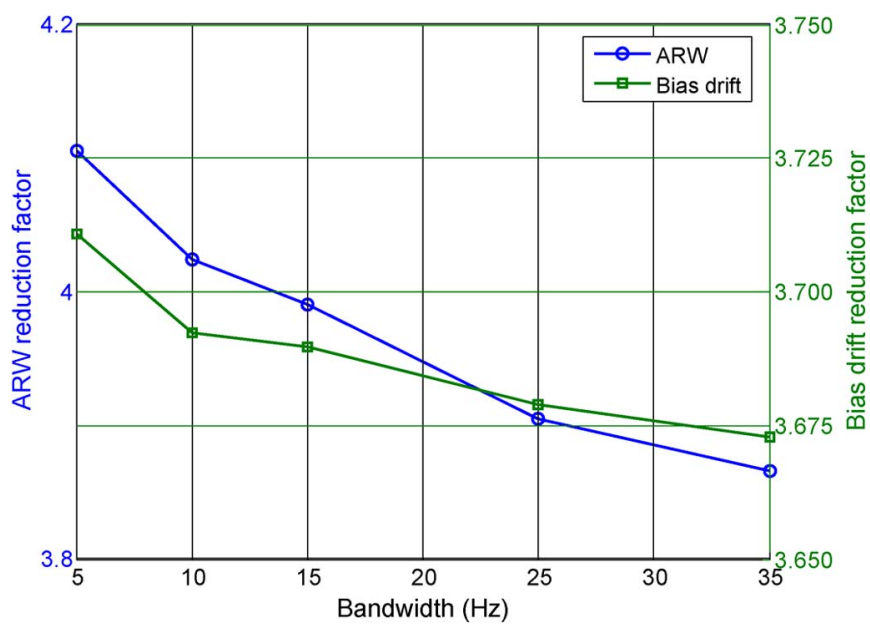

Fig. 12. Plot of Allan variance noise reduction versus the bandwidth of the virtual gyroscope KF.

From Table III, it can be seen that the noise density is reduced by a factor of about 3.67 by the presented KF. In particular, if the Allan variance plot shows that the integration times with respect to the bias instability are identical under the different bandwidth tests, then the bias instability is reduced from $61.99^{\circ} / \mathrm{h}$ to $16.81^{\circ} / \mathrm{h}$, indicating an improvement factor of about 3.7. It is clear that the performance of the presented KF is better than that of a simple averaging process. Additionally, the noise reduction factors of the ARW and bias instability calculated from Table III are shown in Fig. 12; this indicates

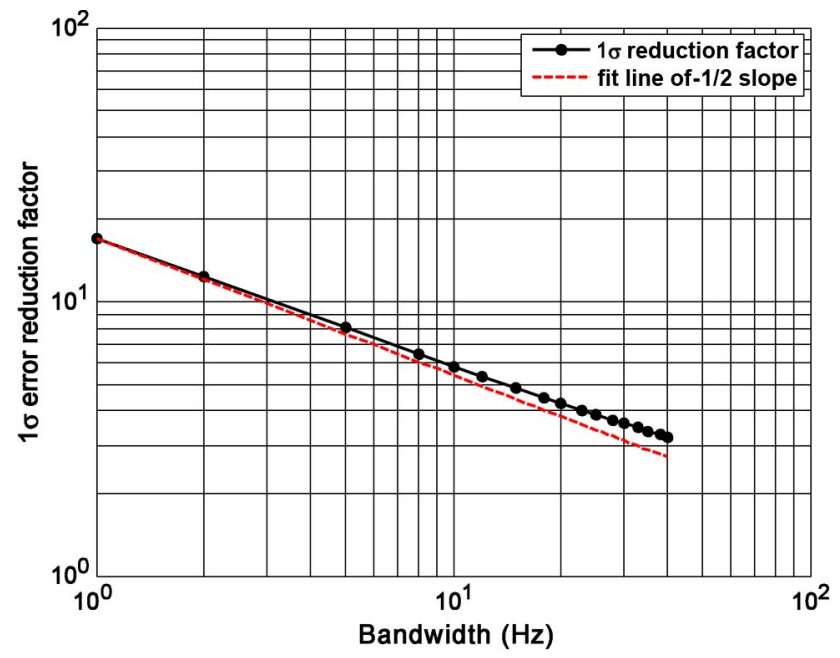

Fig. 13. Plot of the relationship between the $1 \sigma$ error reduction and $\mathrm{KF}$ bandwidth of the virtual gyroscope.

that the reduction factors are increased with decreasing the bandwidth. The noise reduction for the ARW is greater than that for bias instability, which is due to the ARW noise being the dominant noise in the individual gyroscope.

Multiple tests with respect to bandwidth were carried out to further evaluate the noise performance. The $1 \sigma$ errors of a zero rate output recorded for $1 \mathrm{~h}$ before and after Kalman filtering corresponding to the single gyroscope and virtual gyroscope are analyzed. The relationship between the $1 \sigma$ error reduction and $\mathrm{KF}$ bandwidth is shown in Fig. 13, where the noise reduction factor is defined by (21).

From Fig. 13, it can be seen that the $1 \sigma$ reduction factor will increase with decreasing bandwidth. Furthermore, a slope of approximately $-1 / 2$ is observed in the $\log -\log$ plot, which is characteristic of the white noise on an Allan variance plot. Therefore, according to the Allan variance results, it is easy to conclude that the white noise is significantly reduced by choosing the proper $q_{\omega}$ to obtain different bandwidths. It also suggests that the influence of tuning the bandwidth to improve the bias stability is insignificant. As mentioned before, the reason lies in the noise characteristic of an individual gyroscope. If the noise characteristic of the gyroscope changes, then the slope of the fitting line in Fig. 13 will be different.

On the other hand, it is found that the $I F$ follows a power law with the bandwidth as $I F=17.03 \times B W^{-0.4804}$. The RMSE is approximately 0.06 , and $R^{2}=0.9998$. Therefore, we can easily use the power equation to simply trade off bandwidth with accuracy and select an appropriate bandwidth according to the practical application. It should be noted that the aforementioned fitting model is only valid for the presented gyroscope array; if the gyroscope array changes, the model will be different, although the trend will be the same.

\section{CONCLUSION}

In this paper, six identical gyroscopes with a noise density of $0.11^{\circ} / \mathrm{s} \sqrt{ } \mathrm{Hz}$ and a bias instability of $61.99^{\circ} / \mathrm{h}$ have been used to form a virtual gyroscope having a noise density of $0.03^{\circ} / \mathrm{s} \sqrt{ } \mathrm{Hz}$ and a bias instability of $16.81^{\circ} / \mathrm{h}$. This proves that 
the accuracy of MEMS gyroscopes can be improved through combining numerous uncorrelated gyroscopes. With the right conditions, the accuracy improvement is better than that of a simple averaging process.

It needs to be pointed out the narrow bandwidth of the virtual gyroscope will limit the application of the concept, but there do exist some situations where the angular rate changes very slowly, for example, for platform stabilization or cruise control of an airplane. Furthermore, in a dynamic situation requiring a higher bandwidth, the virtual gyroscope can still be used as a simple averaging process.

To obtain the parameter $q_{\omega}$ in real time would be helpful to improve the virtual gyroscope performance and extend the application range of the concept as it would allow adjusting the bandwidth dynamically. Therefore, in future work, an adaptive Kalman filtering approach will be investigated that will regulate $q_{\omega}$ online based on the input signal variance in a specific measurement period.

Another important issue for practical applications of the virtual gyroscope technology is its power consumption since multiple sensors are used. A tradeoff needs to be made between the number of sensors and the available power supply. It is likely that the presented approach is not suitable for batteryoperated or self-powered wireless applications but rather for applications where power consumption is not of primary concern such as in an aircraft.

\section{REFERENCES}

[1] N. El-Sheimy, K.-W. Chiang, and A. Noureldin, "The utilization of artificial neural networks for multisensor system integration in navigation and positioning instruments," IEEE Trans. Instrum. Meas., vol. 55, no. 5, pp. 1606-1615, Oct. 2006.

[2] I. P. Prikhodko, S. A. Zotov, A. A. Trusov, and A. M. Shkel, "Sub-degreeper-hour silicon MEMS rate sensor with 1 million Q-factor," in Proc. Transducers Conf., Beijing, China, Jun. 5-9, 2011, pp. 2809-2812.

[3] F. M. Zaman, A. Sharma, H. Zhili, and F. Ayazi, "A mode-matched silicon-yaw tuning-fork gyroscope with subdegree-per-hour Allan deviation bias instability," J. Microelectromech. Syst., vol. 17, no. 6, pp. 15261536, Dec. 2008.

[4] W. K. Sung, M. Dalal, and F. Ayazi, "A mode-matched 0.9 MHz single proof-mass dual-axis gyroscope," in Proc. Transducers Conf., Beijing, China, Jun. 5-9, 2011, pp. 2821-2824.

[5] G. He and K. Najafi, "A single-crystal silicon vibrating ring gyroscope," in Proc. MEMS, Las Vegas, NV, Jan. 20-24, 2002, pp. 718-721.

[6] S. E. Alper, Y. Temiz, and T. Akin, "A compact angular rate sensor system using a fully decoupled silicon-on-glass MEMS gyroscope," J. Microelectromech. Syst., vol. 17, no. 6, pp. 1418-1429, Dec. 2008.

[7] F. Ayazi and K. Najafi, "A HARPSS polysilicon vibrating ring gyroscope," J. Microelectromech. Syst., vol. 10, no. 2, pp. 169-179, Jun. 2001.

[8] E. Sahin, S. E. Alper, and T. Akin, "Experimental evaluation of alternative drive-mode control electronics developed for high-performance MEMS gyroscopes," in Proc. Transducers Conf., Beijing, China, Jun. 5-9, 2011, pp. 2817-2820.

[9] C. D. Ezekwe and B. E. Boser, "A mode-matching $\Delta \Sigma$ closed-loop vibratory gyroscope readout interface with a $0.004 \% \mathrm{~s} / \mathrm{Hz}$ noise floor over a 50 Hz band," IEEE J. Solid-State Circuits, vol. 43, no. 12, pp. 3039-3048, Dec. 2008.

[10] M. Saukoski, L. Aaltonen, K. Halonen, and T. Salo, "Integrated readout and control electronics for a microelectromechanical angular velocity sensor," in Proc. ESSCIRC, Montreux, Switzerland, Sep. 19-21, 2006, pp. 243-246.

[11] D. S. Bayard and S. R. Ploen, "High accuracy inertial sensors from inexpensive components," U.S. Patent 0187 623, Oct. 2, 2003.

[12] H. L. Chang, L. Xue, W. Qin, G. M. Yuan, and W. Z. Yuan, "An integrated MEMS gyroscope array with higher accuracy output," in Proc. Sensors, 2008 , vol. 8, pp. 2886-2899.
[13] P. A. Stubberud and A. R. Stubberud, "A signal processing technique for improving the accuracy of MEMS inertial sensors," in Proc. ICSENG, Las Vegas, NV, 2008, pp. 13-18.

[14] M. I. Al-Majed and B. N. Alsuwaidan, "A new testing platform for attitude determination and control subsystems: Design and applications," in Proc. IEEE/ASME Int. Conf. Adv. Intell. Mechatron., Singapore, 2009, pp. 1318-1323.

[15] S. C. Stubberud and K. A. Kramer, "Data association for multiple sensor types using fuzzy logic," IEEE Trans. Instrum. Meas., vol. 55, no. 6, pp. 2292-2303, Dec. 2006

[16] IEEE Standard Specification Format Guide and Test Procedure for Coriolis Vibratory Gyros, IEEE Std. 1431, 2004.

[17] Q. M. Lam, T. Hunt, P. Sanneman, and S. Underwood, "Analysis and design of a fifteen state stellar inertial attitude determination system," in Proc. AIAA Conf. Guid. Navig. Control, Austin, TX, 2003, vol. 5483, pp. 11-14.

[18] Q. M. Lam, N. Stamatakos, C. Woodruff, and S. Ashton, "Gyro modeling and estimation of its random noise sources," in Proc. AIAA Conf. Guid. Navig. Control, Austin, TX, 2003, vol. 5562, pp. 1-11.

[19] N. El-Sheimy, H. Y. Hou, and X. J. Niu, "Analysis and modeling of inertial sensors using Allan variance," IEEE Trans. Instrum. Meas., vol. 57, no. 1, pp. 140-149, Jan. 2008.

[20] M. S. Grewal and A. P. Andrews, Kalman Filtering Theory and Practice Using Matlab, 2nd ed. New York: Wiley, 2001, pp. 133-135.

[21] ADXRS300 Data Sheet, 2004. Analog Devices. AD. [Online]. Available: http://www.analog.com/static/imported-files/data_sheets/ADXRS300. pdf

[22] TMS320VC5416 Data Sheet, 2004. Texas Instrum. [Online]. Available: http://focus.ti.com/docs/prod/folders/print/tms320vc5416.html

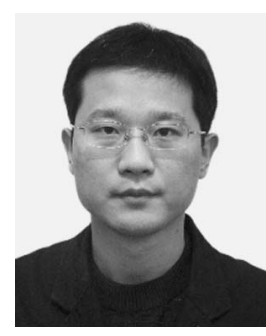

Honglong Chang (M'04) received the B.S., M.S., and Ph.D. degrees in mechanical engineering from Northwestern Polytechnical University (NPU), Xi' an, China, in 1999, 2002, and 2005, respectively.

$\mathrm{He}$ is currently a Professor with the Micro and Nano Electromechanical Systems Laboratory, NPU, where he is also currently the Dean of the Department of Microsystem Engineering. Since September 2011, he has been a Visiting Associate with the Micromachining Laboratory, California Institute of Technology, Pasadena. His research interests include microelectromechanical systems (MEMS) design tools, MEMS inertial sensors, and bio-MEMS.

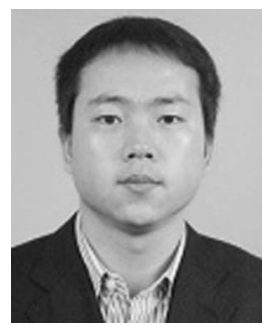

Liang Xue received the M.S. degree in mechanical engineering from Northwestern Polytechnical University, Xi'an, China, in 2008, where he is currently working toward the Ph.D. degree in the Department of Microsystems Engineering.

His current research focuses on microelectromechanical systems inertial sensors and multiple-sensor data-fusion technology.

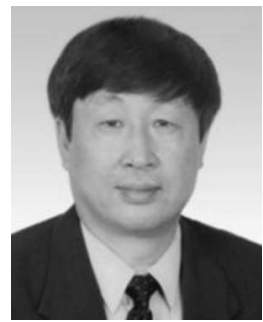

Chengyu Jiang received the Ph.D. degree in mechanical engineering from Nanjing University of Aeronautics and Astronautics, Nanjing, China, in 1988.

$\mathrm{He}$ is currently a Professor with the Department of Microsystem Engineering, Northwestern Polytechnical University, Xi' an, China. His research interests include microsensors and microactuators for aerospace and smart skin for airplane. 


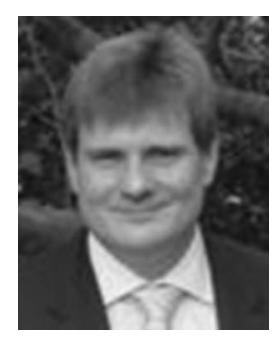

Michael Kraft received the Dipl.-Ing.(Univ.) degree in electrical and electronics engineering from the Friedrich Alexander Universität Erlangen-Nürnberg, Erlangen, Germany, in 1993 and the Ph.D. degree from Coventry University, Coventry, U.K., in 1997, on the development of a microelectromechanical systems (MEMS) accelerometer embedded in a sigma-delta-modulator force-feedback loop.

He is currently a Professor of microsystem technology with the School of Electronics and Computer Science, University of Southampton, Southampton, U.K., where he is also currently the Director of the Southampton Nanofabrication Centre. He then spent two years at the Berkeley Sensors and Actuator Centre, University of California, Berkeley, working on integrated MEMS gyroscopes. Since joining the University of Southampton in 1999, he has focused on novel micro- and nanofabrication techniques, microsensors, and actuators and their interface circuits, particularly for capacitive sensors. He has a broad interest in MEMS and nanotechnology ranging from process development to system integration of MEMS and nanodevices. He has published over 160 peerreviewed journal and conference publications as an author or coauthor. He also contributed to three text books on MEMS and edited a book on MEMS for aerospace and automotive applications.

Dr. Kraft currently serves on several steering and technical committees of international conferences such as IEEE Sensors, Eurosensors, and Micromechanics and Microsystems Europe.

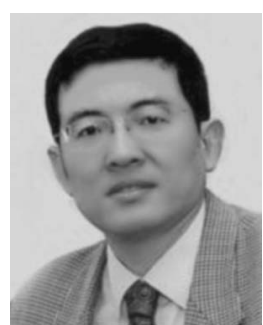

Weizheng Yuan received the Ph.D. degree in mechanical engineering from Northwestern Polytechnical University (NPU), Xi' an, China, in 1996.

$\mathrm{He}$ is currently the Director of the Micro and Nano Electromechanical Systems Laboratory, NPU. His research interests include microsensors, microoptics, and application of microelectromechanical systems. 